\title{
Immunogenicity and protective efficacy of Corsunat a Salmonella Enteritidis sptP mutant as a live attenuated vaccine candidate
}

Zhijie Lin ${ }^{1 \dagger}$, Peipei Tang ${ }^{1 \dagger}$, Yang Jiao ${ }^{1}$, Xilong Kang ${ }^{1}$, Qiuchun $\mathrm{Li}^{1}$, Xiulong $\mathrm{Xu}^{1,3,4}$, Jun Sun ${ }^{1,2}$, Zhiming Pan ${ }^{1 *}$ (D) and Xinan Jiao ${ }^{1 *}$

\begin{abstract}
Background: Salmonella enterica serovar Enteritidis (S. Enteritidis) is a highly adaptive pathogen in both humans and animals. As a Salmonella Type III secretion system (T3SS) effector, Salmonella protein tyrosine phosphatase (SptP) is critical for virulence in this genus. To investigate the feasibility of using C50336 $\Delta$ sptP as a live attenuated oral vaccine in mice, we generated the sptP gene deletion mutant C50336 $\Delta$ sptP in S. Enteritidis strain C50336 by $\lambda$-Red mediated recombination and evaluated the protective ability of the $S$. Enteritidis sptP mutant strain C50336 AsptP against mice salmonellosis.
\end{abstract}

Results: We found that C50336 $\Delta$ sptP was a highly immunogenic, effective, and safe vaccine in mice. Compared to wild-type C50336, C50336 AsptP showed reduced virulence as confirmed by the $50 \%$ lethal dose $\left(L_{50}\right)$ in orally infected mice. C50336 ssptP also showed decreased bacterial colonization both in vivo and in vitro. Immunization with C50336 $\Delta$ sptP had no significant effect on body weight and did not result in obvious clinical symptoms relative to control animals treated with phosphate-buffered saline (PBS), but induced humoral and cellular immune responses at 12 and 26 days post inoculation. Immunization with $1 \times 10^{8}$ colony-forming units (CFU) C50336 $\Delta$ sptP per mouse provided 100\% protection against subsequent challenge with the wild-type C50336 strain, and immunized mice showed mild and temporary clinical symptoms as compared to those of control group.

Conclusions: These results demonstrate that C50336 AsptP can be a live attenuated oral vaccine for salmonellosis.

Keywords: Salmonella Enteritidis, SptP, Vaccine, Immunogenicity, Immune protection

\section{Background}

Salmonella spp. is a Gram-negative, facultative anaerobe and intracellular pathogen in both humans and animals. Infection by Salmonella is a major public health problem [1], causing an estimated 93.8 million illnesses and 155,000 deaths each year worldwide [2] and more than 1 million illnesses and 350 deaths each year in the U.S. [3]. In the past 20 years, Salmonella enterica serovar Enteritidis ( $S$. Enteritidis) has been one of the most common

\footnotetext{
* Correspondence: zmpan@yzu.edu.cn; jiao@yzu.edu.cn

${ }^{\dagger}$ Equal contributors

'Jiangsu Key Laboratory of Zoonosis, Jiangsu Co-Innovation Center for Prevention and Control of Important Animal Infectious Diseases and Zoonosis, MOA Key Laboratory of Prevention and Control of Biological Hazard Factors (Animal Origin) for Agrifood Safety and Quality, MOE Joint International Research Laboratory of Agriculture and Agri-product Safety, Yangzhou University, Yangzhou 225001, China

Full list of author information is available at the end of the article
}

serotypes in salmonellosis in humans despite the implementation of control and prevention measures [4]. Humans can be infected with Salmonella via consumption of contaminated pork, beef, poultry, and eggs or contact with fecal matter in places with poor sanitation. Salmonellosis in humans is characterized by abdominal pain, diarrhea, nausea, vomiting, fever, and headache [5].

Salmonellosis treatment and protection strategies include antimicrobial therapy and vaccination, but emergence of multidrug-resistant strains is becoming a serious global problem [6, 7]. Vaccines based on inactivated bacteria can potentially prevent salmonellosis [8]; however, attenuated live vaccines generated by deletion of various identified Salmonella virulence genes have higher immunogenicity and greater efficacy than killed bacteria [9-14]. 
Salmonella protein tyrosine phosphatase (SptP) is a Salmonella T3SS effector protein encoded in Salmonella pathogenicity islands (SPI)-1. The SptP protein has an $\mathrm{N}$-terminal domain that acts as a GTPase-activating protein for Cdc42 and Rac1, mediating alterations in the actin cytoskeleton of host cells [15], as well as a Cterminal domain that inhibits mitogen-activated protein kinase and extracellular signal-regulated kinase signaling [16]. SptP also suppresses interleukin-8 (IL-8) production and consequently the inflammatory response in hosts, thereby promoting Salmonella invasion and intracellular replication $[17,18]$. In a mouse infection model, SptP suppressed the degranulation of mast cells and blocked neutrophil recruitment [19].

In this study, we generated the sptP gene deletion mutant C50336 $\Delta$ sptP in S. Enteritidis strain C50336 by $\lambda$-Red mediated recombination [20]. The growth characteristics of C50336 $\Delta$ sptP were similar to those of wildtype C50336. We therefore investigated the feasibility of using C50336 $\Delta p t P$ as a live attenuated oral vaccine in mice by evaluating virulence, changes in body weight and clinical symptoms, bacterial persistence, immune responses, and protective efficacy.

\section{Methods}

\section{Bacterial strains and cells lines}

The wild-type $S$. Enteritidis strain C50336 was obtained from the National Institute for the Control of Pharmaceutical and Biological Products (Beijing, China). The sptP deletion mutant strain C50336 $\Delta$ sptP was constructed by $\lambda$-Red-mediated recombination as previously described [20]. Briefly, the sequence of the chloramphenicol resistance cassette $\left(\mathrm{Cm}^{\mathrm{R}}\right)$ was amplified from plasmid pKD3, including 39-bp homology extensions at the $5^{\prime}$ and $3^{\prime}$ ends of the sptP gene (primers: forward $5^{\prime}$-tgaatcagcaggaagtgctcaaaaacatactgcaggaatgtgtaggctggagctgcttc $-3^{\prime}$; reverse $5^{\prime}$-cttactttcagatagttctaaaagtaagctatgtttttaatgggaattagccatggtcc $\left.-3^{\prime}\right)$. PCR products were purified and transferred into C50336 cells containing plasmid pKD46 by electroporation. Recombinant C50336-Cm ${ }^{\mathrm{R}}$ cells grown on Luria-Bertani (LB) agar plates were selected for both $\mathrm{Cm}^{\mathrm{R}}$ and ampicillin resistance $\left(A m p^{R}\right)$. Allelic replacement of $s p t P$ with the $\mathrm{Cm}$ cassette was verified by PCR analysis (primers: forward $5^{\prime}$-atccgaactactttacgc- $3^{\prime}$; reverse $5^{\prime}$-tgaatggtattctactgg$\left.3^{\prime}\right)$ and DNA sequencing. The cassette was then excised by introducing the Flp recombinase-expressing vector pCP20. Biochemical tests were performed using the API 20E identification kit (BioMérieux, Lyon, France) and VITEK 2 Gram-negative bacilli test (BioMérieux) according to the manufacturer's protocol. Bacteria were cultured in LB broth followed by overnight incubation at $37{ }^{\circ} \mathrm{C}$ with shaking at $180 \mathrm{rpm}$ [21]. XLT4 (Difco Laboratories, San Jose, CA, USA) and 1.5\% LB agar were used for bacterial culture and counts of colony-forming units (CFU).

Human epithelial cells Caco-2 BBE and mouse macrophage RAW264.7 cells were cultured and maintained in Dulbecco's modified Eagle's medium (DMEM) containing $10 \%$ fetal bovine serum (FBS), $50 \mu \mathrm{g} / \mathrm{ml}$ streptomycin, and $50 \mathrm{U} / \mathrm{ml}$ penicillin.

\section{Experimental animals}

Female BALB/c mice ( 8 weeks old) used for vaccination experiments were obtained from the Comparative Medical Center of Yangzhou University (Yangzhou, China). The animal experiments were all approved by the Animal Care and Ethics Committee of Yangzhou University, Yangzhou, China (Approval ID: SYXK [Su] 2012-0029).

\section{Assessment of bacterial virulence}

The virulence of mutant C50336 $\Delta$ sptP and wild-type strain C50336 was evaluated in BALB/c mice by oral inoculation of the mice with various doses of the bacterial strains. The morbidity and mortality of the mice were observed as previously described [22]. Briefly, water and food were withdrawn $4 \mathrm{~h}$ before oral gavage with $100 \mu \mathrm{l}$ of $5 \%$ sodium bicarbonate to neutralize stomach acid; $1 \mathrm{~h}$ later, mice were administered 10-fold dilutions of C50336 $\Delta$ sptP or C50336 $\left[1 \times 10^{8}-1 \times 10^{4} \mathrm{CFU}\right.$ in $100 \mu \mathrm{l}$ of phosphate-buffered saline (PBS)] by oral gavage. Control mice received $100 \mu \mathrm{l}$ of PBS via the same route. All deaths of mice were recorded over the 16-day experimental period. The $\mathrm{LD}_{50}$ was calculated using the Karber and Behrens method [23].

\section{Bacterial colonization in cells}

Human epithelial cells Caco-2 BBE and mouse macrophage RAW264.7 were grown in DMEM with 10\% FBS. At $90-100 \%$ confluence, the monolayers were washed three times with PBS, and then the cells were colonized with an equal number of the indicated bacteria for 30 min (multiplicity of infection $=100$ ). For bacterial adhesion, the cells were washed, and then incubated with PBS containing Triton X-100 (0.5\%) at $37{ }^{\circ} \mathrm{C}$ for $10 \mathrm{~min}$. For bacterial invasion, $30 \mathrm{~min}$ after bacterial colonization, the cells were incubated for an additional $30 \mathrm{~min}$ in DMEM with gentamicin $(100 \mu \mathrm{g} / \mathrm{ml})$, washed and incubated with PBS containing Triton X-100 (0.5\%) at $37{ }^{\circ} \mathrm{C}$ for $10 \mathrm{~min}$ [24]. Serial 10 -fold dilutions of cell lysates were plated on XLT4 agar and incubated at $37{ }^{\circ} \mathrm{C}$ for $12-16 \mathrm{~h}$. The number of bacteria was counted and expressed as $10^{2} \mathrm{CFU} / \mathrm{ml}$.

\section{Bacterial colonization and persistence in organs and tissues}

Bacterial colonization and persistence in the internal organs of infected mice were evaluated. Mice were 
administered $1 \times 10^{8}$ or $1 \times 10^{7} \mathrm{CFU}$ of C50336 $\Delta p t P$ by oral gavage, and six mice per day were sacrificed at 1 , $3,5,7,14,21,28$, and 44 days post-immunization (DPI). Liver, spleen, and mesenteric lymphadenitis (mLN) samples were aseptically collected, weighed, and homogenized in $1 \mathrm{ml}$ of PBS; Serial 10-fold dilutions of tissue homogenates $(100 \mu \mathrm{l}$ each) were plated on XLT4 agar and incubated at $37{ }^{\circ} \mathrm{C}$ for $12-16 \mathrm{~h}$. Bacteria were counted and the numbers are expressed as $\log _{10} \mathrm{CFU} / \mathrm{g}$.

\section{Changes in body weight and clinical symptoms after immunization}

Mice were immunized with C50336 $\Delta p t P$ at $1 \times 10^{8}$ or $1 \times 10^{7} \mathrm{CFU}$ in $100 \mu \mathrm{l}$ of PBS by oral gavage (two doses of immunization at 0 and 14 DPI). Control animals received $100 \mu \mathrm{l}$ of PBS. Body weight was recorded at 1, 3, 5, 7, 14, 21, 28, and 44 DPI. The mice were monitored from 1 to 44 DPI for clinical symptoms, including feed intake, susceptibility, depression and diarrhea.

\section{Serum IgG test}

Specific IgG antibody and IgG subtype levels were measured by enzyme-linked immunosorbent assay (ELISA) using soluble antigens prepared from $S$. Enteritidis strain C50336 (SEAgP) as the coating antigen in 96-well plates $(100 \mu \mathrm{l}$ of SEAgP at $2 \mu \mathrm{g} / \mathrm{ml}$ in each well). Serum samples were collected from mice at 12 and 26 DPI and diluted 1:50 for use as the primary antibody. Horseradish peroxidase (HRP)-conjugated goat anti-mouse IgG (or anti-mouse IgG1 and IgG2a for IgG subtype detection) was used as the secondary antibody (dilution 1:10,000). HRP activity was determined using 3,3',5,5' -tetramethylbenzidine (Sigma-Aldrich, St. Louis, MO, USA). Absorbance was read at $450 \mathrm{~nm}$ using an automated microplate reader (Titertek Multiskan; Flow Laboratories, Lugano, Switzerland).

\section{Lymphocyte stimulation test}

The lymphocyte proliferation assay was performed using SEAgP as a stimulator. The spleen was isolated from the mice at 12 and 26 DPI and homogenized. Splenic lymphocytes were obtained by passing the homogenate through a filter with $40-\mu \mathrm{m}$ pores (BD Biosciences, San Jose, CA, USA). Cell viability was determined based on Trypan Blue dye exclusion. Spleen mononuclear cell suspensions $\left(1 \times 10^{7}\right.$ cells $\left./ \mathrm{ml}\right)$ were cultured in Roswell Park Memorial Institute 1640 medium containing 10\% FBS, $50 \mu \mathrm{g} / \mathrm{ml}$ streptomycin, and $50 \mathrm{U} / \mathrm{ml}$ penicillin in 96-well tissue culture plates with $10 \mu \mathrm{g} / \mathrm{ml} \mathrm{SEAgP} \mathrm{or}$ $10 \mu \mathrm{g} / \mathrm{ml}$ concanavalin A (Con A, a lymphocyte mitogen) as a positive control at $37{ }^{\circ} \mathrm{C}$ in a humidified atmosphere of $5 \% \mathrm{CO}_{2}$ for $72 \mathrm{~h}$. Lymphocyte proliferation was measured with a BrdU kit (Roche, Basel, Switzerland). Blastogenic responses to SEAgP are expressed as a mean stimulation index (SI) calculated based on the optical density of stimulated cultures at $450 \mathrm{~nm}$, as previously described $[25,26]$.

\section{Immune protection assessment of C50336 $\Delta$ sptP}

The protective efficacy of the mutant C50336 $\Delta$ sptP was evaluated in mice immunized orally with $1 \times 10^{8} \mathrm{CFU}$ (group A) in $100 \mu \mathrm{l}$ of PBS (two doses of immunization at 0 and $14 \mathrm{DPI}$ ). Control mice (groups B and C, respectively) received $100 \mu \mathrm{l}$ of PBS. At 28 DPI, group A was challenged orally with $1 \times 10^{8} \mathrm{CFU}$ of wild-type strain C50336 in $100 \mu \mathrm{l}$ of PBS, whereas group B was challenged with $5 \times 10^{5}$ CFU of wild-type strain C50336 in the same way. Group $\mathrm{C}$ received $100 \mu \mathrm{l}$ of PBS as a blank control. The number of surviving mice and extent of bacterial colonization in internal organs were determined at 16 days post challenge. The number of CFUs recovered from tissues was replica-verified by PCR analysis (Primers: Forward 5' -atccgaactactttacgc3'; Reverse 5'-tgaatggtattctactgg-3'). Clinical symptoms, including anorexia, diarrhea, depression, and mortality, were recorded daily.

\section{Statistical analysis}

Data are expressed as the mean \pm SEM. All statistical tests were two-sided. $P<0.05$ was considered to be statistically significant. Differences between two samples were evaluated using Student's $t$ test. Statistical analyses were performed using SAS v.9.4 software (SAS Institute, Cary, NC, USA).

\section{Results}

\section{Construction of a sptP deletion mutant $C 50336 \Delta$ sptP in S. Enteritidis}

The sptP gene deletion mutant was established in $S$. Enteritidis strain $C 50336$ by $\lambda$-Red-mediated recombination. Our PCR data showed that the sptP gene was deleted in the C50336 $\Delta$ sptP mutant (Fig. 1a). The growth characteristics of the C50336 $\Delta$ sptP mutant and wildtype C50336 cells were determined in LB liquid medium, with no significant difference observed between the mutant and wild type (Fig. 1b). Biochemical tests were performed using the API 20E identification kit (BioMérieux, Lyon, France) and VITEK 2 Gramnegative bacilli test (BioMérieux) according to the manufacturer's protocol, also showed no difference between these strains (data not shown).

\section{C50336 $\Delta$ sptP exhibits reduced virulence in a murine model}

The virulence of $S$. Enteritidis wild-type C50336 and C50336 $\operatorname{sptP}$ was evaluated in BALB/c mice with oral gavage. As shown in Table 1 , the $\mathrm{LD}_{50}$ of $\mathrm{C} 50336$ was 


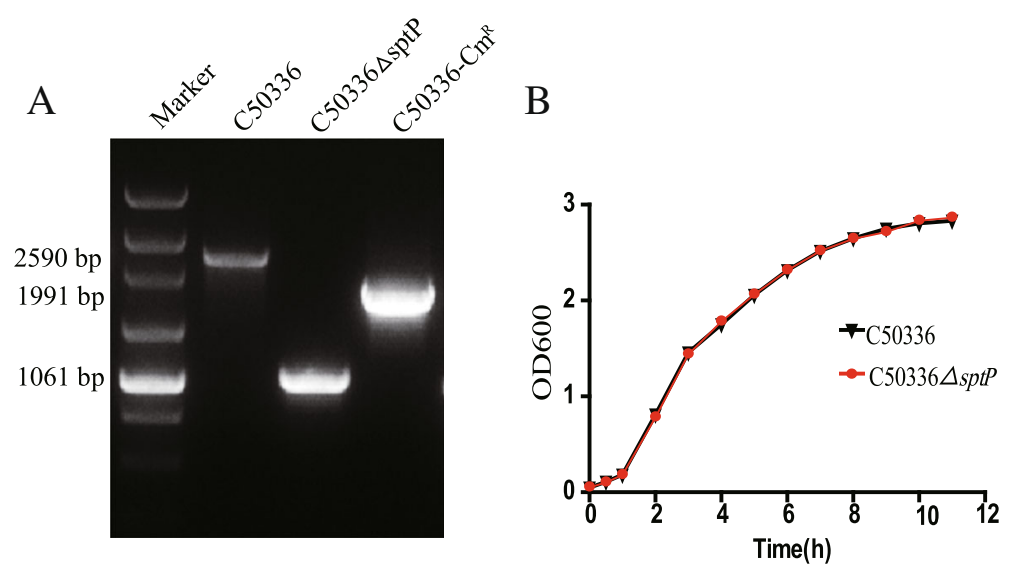

Fig. 1 Construction of sptP deletion mutant in S. Enteritidis strain C50336. a PCR verification of C50336 $\Delta$ sptP and C50336-Cm . The wild type strain harbors the complete sptP gene, with a PCR product length of $2509 \mathrm{bp}$, whereas the PCR product from C50336 $\Delta$ sptP has a length of $1061 \mathrm{bp}$, and the product from the C50336-Cm ${ }^{\mathrm{R}}$ has a length of $1991 \mathrm{bp}$ (right). b Growth curves of wild-type S. Enteritidis C50336 and C50336 AsptP. Bacteria were grown in liquid LB medium at $37^{\circ} \mathrm{C}$ for $12 \mathrm{~h}$ with agitation, and the $\mathrm{OD}_{600}$ values of triplicate cultures in LB medium were determined in 1-h intervals

$3.16 \times 10^{5} \mathrm{CFU}$, but no mice died in the group challenged by $C 50336 \Delta s p t P$, indicating that the virulence of the $S$. Enteritidis mutant C50336 $\Delta$ sptP was attenuated.

\section{sptP deletion leads to reduced bacterial colonization in cells}

The bacterial colonization in cells was determined in human epithelial Caco-2 BBE and mouse macrophage RAW264.7 cells. As shown in Fig. 2, the total number of associated bacteria was not different between C50336 $\Delta s p t P$ and C50336 in Caco-2 BBE and RAW264.7 cells. However, cells colonized with C50336 $\Delta s p t P$ showed a decreased intracellular bacterial load compared to cells colonized with C50336 (Fig. 2a \& b). These results indicate that $S$. Enteritidis with sptP deletion has reduced bacterial colonization in vitro.

Table $1 \mathrm{LD}_{50}$ of $\mathrm{C} 50336 \Delta$ sptP in BALB/c mice

\begin{tabular}{llll}
\hline Strain & $\begin{array}{l}\text { Inoculation } \\
\text { dose (CFU) }\end{array}$ & $\begin{array}{l}\text { Number of dead mice/total } \\
\text { number of mice }\end{array}$ & $L_{50}$ \\
\hline C50336 & $1.0 \times 10^{7}$ & $5 / 5$ & $3.16 \times 10^{5}$ \\
& $1.0 \times 10^{6}$ & $4 / 5$ & \\
& $1.0 \times 10^{5}$ & $1 / 5$ & \\
& $1.0 \times 10^{4}$ & $0 / 5$ & - \\
C50336 sptP & $1.0 \times 10^{8}$ & $0 / 5$ & \\
& $1.0 \times 10^{7}$ & $0 / 5$ & \\
& $1.0 \times 10^{6}$ & $0 / 5$ & \\
& $1.0 \times 10^{5}$ & $0 / 5$ & \\
& $1.0 \times 10^{4}$ & $0 / 5$ & \\
\hline
\end{tabular}

Colonization and persistence of Salmonella C50336 $\Delta$ sptP in internal organs and tissues

The bacterial number was calculated in the liver, spleen and $\mathrm{mLN}$ of $\mathrm{BALB} / \mathrm{c}$ mice following oral immunization with $1 \times 10^{8} \mathrm{CFU}$ or $1 \times 10^{7} \mathrm{CFU}$ C50336 $\Delta s p t P$. As shown in Fig. 3, Salmonella colonization reached the highest level at 3 DPI (first immunization) in the liver, spleen, and mLN (Fig. 3b-d), and at 28 DPI (14 days post second immunization), two and one of the six mice were positive for Salmonella in the liver and spleen, respectively, in both the $1 \times 10^{8}$ and $1 \times 10^{7} \mathrm{CFU}$ - immunized groups. In the $1 \times 10^{7} \mathrm{CFU}$ immunized group, Salmonella was detected in the mLN of one mouse. At 44 DPI, we did not detect Salmonella in any tissues from immunized mice. All samples from the negative control group (PBS group) were negative for Salmonella.

\section{Immunogenicity of C50336 $\Delta$ sptP}

The changes in body weight and clinical symptoms were monitored in mice following immunization with C50336 $\Delta$ sptP. The body weights of the mice were shown in Fig. 3a. A decrease in body weight loss was noticed in the initial days of post immunization, but no statistically significant differences were observed among the three groups (immunized with $1 \times 10^{8} \mathrm{CFU}$ C50336 $\Delta p t P$, immunized with $1 \times 10^{7} \mathrm{CFU}$ C50336 $\Delta$ sptP, and treated with PBS as a control). No significant differences were found in clinical symptoms among the three groups, although slight and temporary anorexia was observed in vaccinated mice. Clinical signs of disease such as lethargy and diarrhea were absent in all immunized mice and control mice. The procedures for immunization are shown in Fig. 4a. 

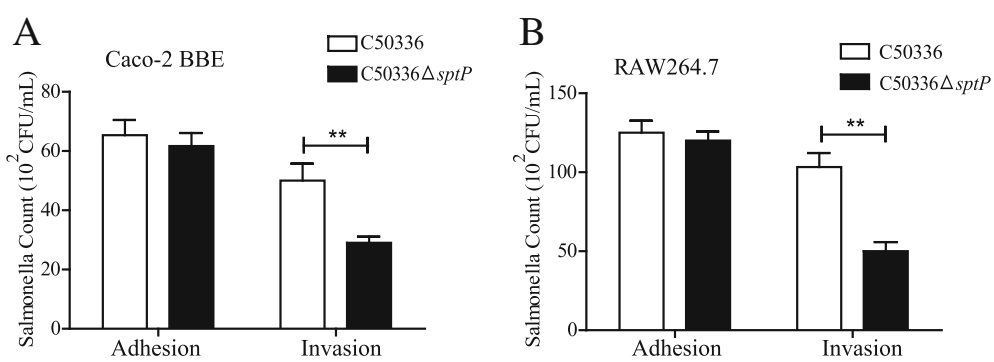

Fig. 2 Bacterial colonization in cells. Salmonella adhesion and invasion in the Human epithelial Caco-2 BBE cells (a), and mouse macrophage RAW264.7 cells (b). The number of Salmonella was determined and presented as $10^{2} \mathrm{CFU} / \mathrm{mL}$. Data are expressed as the mean \pm SEM. ${ }^{* *} P \leq 0.01$

The humoral immune responses were evaluated in mice following oral immunization with C50336 $\Delta p t P$. Serum IgG levels were determined by indirect ELISA. As shown in Fig. 4b, mice immunized with $1 \times 10^{8} \mathrm{CFU}$ and $1 \times 10^{7} \mathrm{CFU}$ of C50336 $\Delta$ sptP showed significantly higher levels of serum IgG at 12 and 26 DPI than the control group. Furthermore, the titers of the IgG1 subtype were significantly higher than those of IgG2a, demonstrating that $S$. Entertidis C50336 $\Delta$ sptP tends to induce a Th2 immune response in mice (Fig. 4c).

The cellular immune responses in mice following oral immunization with C50336 $\Delta$ sptP were evaluated by splenic lymphocyte proliferation assay. As shown in Fig. 4d, the SI values of immunized mice were significantly higher than control mice after stimulation with SEAgP or lymphocyte mitogen ConA at 12 and 26
DPI. This finding indicates that C50336 $\Delta s p t P$ also induces cellular immune responses in mice.

\section{C50336 $\Delta$ sptP protects mice against oral challenge with wild-type $S$. Enteritidis}

Mice were orally vaccinated with 2 doses of $1 \times 10^{8} \mathrm{CFU}$ of C50336 $\Delta$ sptP, and then at $28 \mathrm{DPI}$, they were challenged with wild-type C50336. The percentage of surviving mice at 16 days post challenge is shown in Table 2. None of the immunized mice died in group A and group $\mathrm{C}$, whereas ten of fifteen mice died in control group B. Slight and temporary anorexia as well as depression were observed following challenge in the immunized mice (group A) when compared the blank control group C. Severe clinical symptoms and mortality
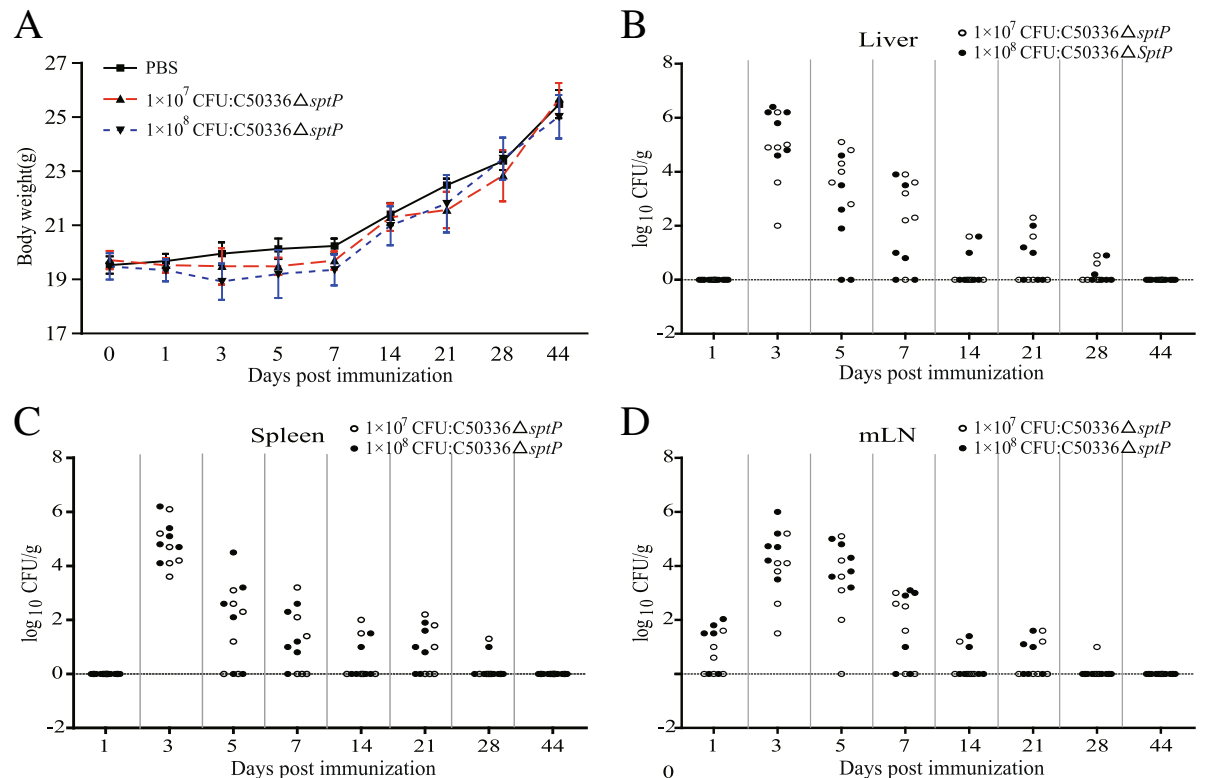

Fig. 3 Body weight (a) and bacterial colonization in mouse organs post immunization. Salmonella colonization and persistence in the liver (b), spleen $(\mathbf{c})$, and $\mathrm{mLN}(\mathbf{d})$ of mice following oral immunization with $1 \times 10^{7}$ CFU or $1 \times 10^{8}$ CFU of C50336 $\Delta$ sptP. Control mice received $100 \mu$ l of PBS and were negative for Salmonella in the liver, spleen, and mLN. The number of Salmonella was determined and represented as Log ${ }_{10}$ CFU/g 
A

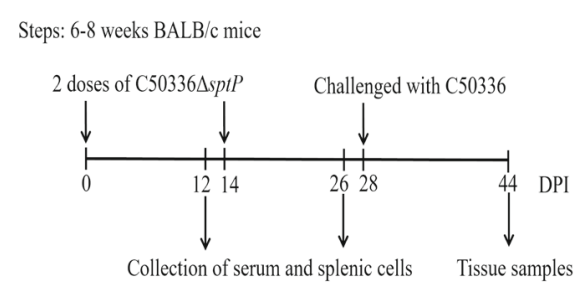

C

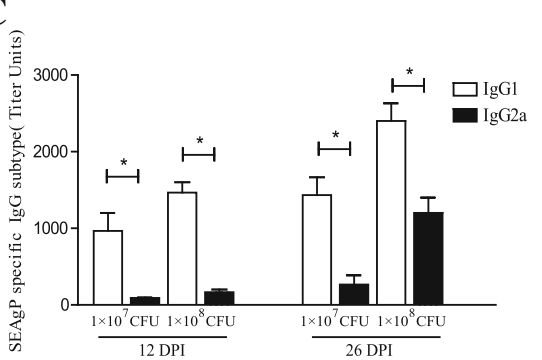

B

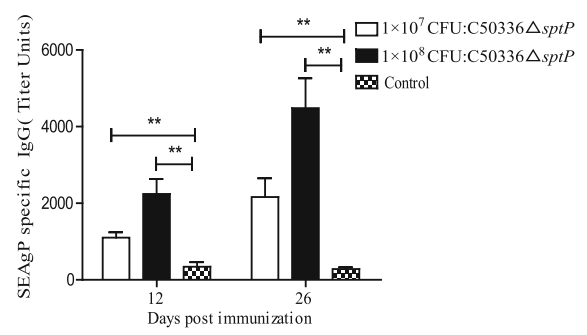

D

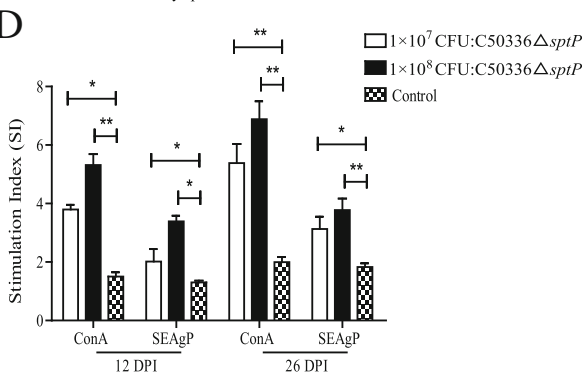

Fig. 4 Immunogenicity of C50336 $\Delta$ sptP. Procedure for immunization (a). Determination of serum lgG levels (b) and lgG subtype levels (c) at 12 and 26 days post immunization. Stimulation index (SI) of mice lymphocytes determined by splenic lymphocyte proliferation assay using soluble antigen SEAgP $(\mathbf{d})$ or lymphocyte mitogen ConA as a positive control. The vaccinated group received $1 \times 10^{7}$ CFU or $1 \times 10^{8}$ CFU of C50336 $\Delta$ sptP orally, and the control group received $100 \mu \mathrm{l}$ of PBS. Data are expressed as the mean \pm SEM. ${ }^{*} P \leq 0.05$, ${ }^{* *} P \leq 0.01$

were observed following challenge in the nonimmunized mice. The survival curve is shown in Fig. 5a.

All surviving mice were sacrificed at 16 days post challenge, and tissues including the liver, spleen and cecum were sampled. As shown in Fig. 5, for the group vaccinated with $1 \times 10^{8} \mathrm{CFU}$ of C50336 $\Delta$ sptP, four of ten immunized mice carried a low level of Salmonella in the liver and spleen (Fig. 5b \& c), and two of ten mice were positive for Salmonella in the cecum (Fig. 5d). The mice in group B carried a higher level of Salmonella in the liver, spleen, and cecum than the immunized mice from group A. All samples from the blank control group (group C) were negative for Salmonella.

\section{Discussion}

In current study, we constructed an sptP mutant in $S$. Enteritidis C50336, and evaluated the efficacy of C50336 $\Delta$ sptP as a candidate live attenuated vaccine for Salmonella infection based on virulence, changes in body weight and clinical symptoms, bacterial colonization, serum IgG level, splenic lymphocyte proliferation and protective efficiency in mice. Our results showed that the body weight change of mice orally vaccinated with $\mathrm{C} 50336 \Delta \operatorname{sptP}\left(1 \times 10^{7} \mathrm{CFU}\right.$ or $\left.1 \times 10^{8} \mathrm{CFU}\right)$ was similar to that of the PBS control. After immunization with C50336 $\Delta s p t P$, only small amounts of Salmonella colonized and persisted in the liver and spleen of the mice at 28 days, but strong humoral and cellular immune responses were induced to protect the mice against secondary $S$. Enteritidis challenge.

Salmonella enterica subsp. enterica includes several important serovars including Typhimurium, Enteritidis Typhi, and Paratyphi, which are the leading sources of human salmonellosis. Vaccines have been developed for one of these serovars and will prevent disease caused by $S$. Typhimurium or $S$. Enteritidis [27]. Symptoms of human Salmonellosis include abdominal pain, diarrhea, nausea, vomiting, fever, and headache [5]. S. Enteritidis is also increasingly reported in cases of invasive and extra-intestinal infections, such as arthritis, septicemia, meningitis, endocarditis, and urinary tract infections [28-34]. Infection with Salmonella is usually caused by consumption of contaminated pork and beef, poultry and eggs. It is necessary to take effective measures to

Table 2 Protective effects of C50336 $\Delta$ sptP in mice

\begin{tabular}{|c|c|c|c|c|c|c|c|c|}
\hline \multirow[t]{2}{*}{ Group } & \multicolumn{2}{|l|}{ Vaccination } & \multirow[t]{2}{*}{ Number } & \multicolumn{3}{|c|}{ Challenge } & \multirow[t]{2}{*}{ Survivors/total } & \multirow{2}{*}{$\begin{array}{l}\text { Surviva } \\
\text { rate }(\%\end{array}$} \\
\hline & Strain & Dose (CFU) & & Strain & Route & Dose (CFU) & & \\
\hline A & C50336 $\Delta$ sptP & $1 \times 10^{8}$ & 10 & C50336 & oral & $1 \times 10^{8}$ & $10 / 10$ & 100 \\
\hline B & PBS & - & 10 & C50336 & oral & $5 \times 10^{5}$ & $5 / 15$ & 33 \\
\hline C & PBS & - & 10 & PBS & oral & - & $5 / 5$ & 100 \\
\hline
\end{tabular}



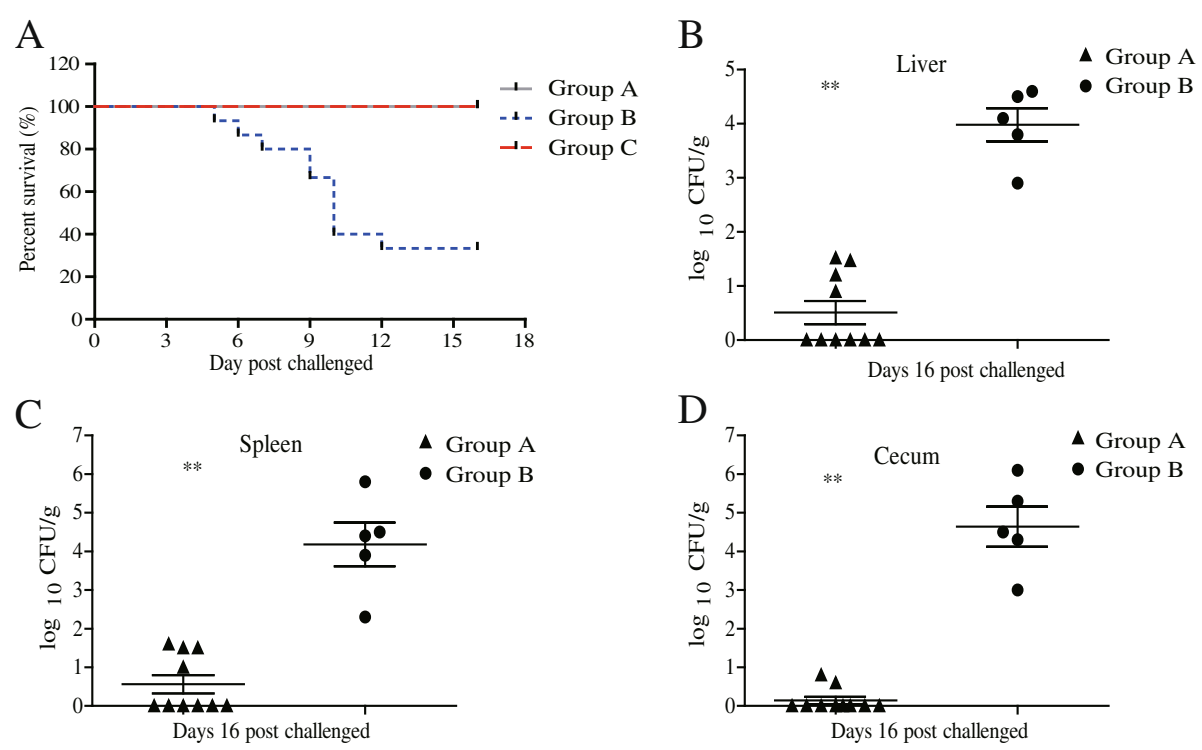

Fig. 5 Survival curve (a) and bacterial colonization in challenged mice. Bacterial colonization in the liver (b), spleen (c) and cecum (d) of challenged mice (Table 2, group A, immunized with C50336 $\Delta$ sptP and then challenged with $1 \times 10^{8}$ CFU of C50336; group B, immunized with PBS and then challenged with $5 \times 10^{5}$ CFU of C50336). All samples from the blank control group were negative for Salmonella (Table 2, group C). The number of bacteria was determined and expressed as $\log _{10}$ CFU/g. Data are expressed as the mean \pm SEM, **P $\leq 0.01$

control and prevent Salmonella infection. Vaccination with live attenuated Salmonella may be a viable choice. Many live attenuated vaccines have been developed against Salmonella and have been confirmed to be generally more effective than vaccines prepared with dead bacteria [9-14].

The ideal vaccine should be avirulent, especially for live attenuated vaccines. Some $S$. Typhimurium and $S$. Enteritidis mutants with deletion of T3SS effector encoding genes or SPI-1 or SPI-2 display decreased virulence in mice, poultry, pigs, and humans [35-40]. In the current study, the $\mathrm{LD}_{50}$ of C50336 $\Delta$ sptP in mice inoculated by the oral route demonstrated that the virulence of the mutant C50336 $\Delta s p t P$ was significantly decreased in comparison with that of the wild-type strain. The body weight of mice immunized with C50336 $\Delta$ sptP was not significantly different from that of control mice and no or less clinical symptoms were observed following immunization. All of these findings show that C50336 $\Delta$ sptP has almost no side effects in terms of growth performance in mice.

For live attenuated Salmonella vaccines, it is critical to stimulate the humoral and cellular immune responses in the immunized host [8, 41]. Systemic dissemination of Salmonella can lead to antigen presentation, resulting in high induction of specific humoral and cellular immune responses, and efficient protection against secondary infection [12, 42]. In this study, the Salmonella-specific serum IgG level in mice immunized with C50336 $\Delta$ sptP at 12 and 26 DPI was significantly higher than the antibody level detected in the control group. In addition, the SI values of splenic lymphocyte proliferation in immunized mice were significantly higher than that in control mice. All of these findings indicate that strong humoral and cellular immune responses can be stimulated by $C 50336 \Delta s p t P$. In addition, the mice vaccinated with $1 \times 10^{8}$ CFU C50336 $\Delta$ sptP showed higher levels of serum IgG and splenic lymphocyte proliferation capability than mice vaccinated with $1 \times 10^{7}$ CFU C50336 $\Delta s p t P$.

$S$. Enteritidis deleted with one or several effector encoding genes provided efficacious protection against secondary $S$. Enteritidis challenge in mice $[12,13]$. In our study, to evaluate the protective efficacy of C50336 $\Delta$ sptP, we orally immunized mice with $1 \times 10^{8} \mathrm{CFU}$ of C50336 $\Delta$ sptP, and determined protection rates following oral challenge with $1 \times 10^{8} \mathrm{CFU}$ of wild-type C50336. Our results show that mice immunized with $1 \times 10^{8} \mathrm{CFU}$ of C50336 $\Delta$ sptP developed extensive humoral and cellular immune responses and experienced $100 \%$ protection against subsequent $S$. Enteritidis challenge.

Pathogenesis of Salmonella is associated with a specialized ability to invade and persist within intestinal epithelial cells, where it can replicate and evade the host immune system [43]. T3SS-1 and T3SS-2 are encoded by SPI-1 and SPI-2 respectively, secreting various effector proteins that are essential for Salmonella colonization and intracellular persistence in the host $[11,35,44]$. SptP is a T3SS-1 effector protein that mediates the reversion of pathogen-induced changes in the actin cytoskeleton after Salmonella invasion 
[15]. In our study, S. Enteritidis C50336 with deletion of sptP led to decreased invasion in Caco-2 BBE and RAW264.7 cells. SptP also inhibits the MAPK pathway, suppresses IL-8 production, and reduces the inflammatory response in host cells, thereby enhancing intracellular Salmonella replication [16-18]. In current study, deletion of sptP resulted in induction of strong humoral and cellular immune responses against subsequent Salmonella challenge.

\section{Conclusion}

Our present work demonstrates that vaccination of mice with the candidate vaccine C50336 $\Delta$ sptP conferred development of acquired immunity and efficacious protection against experimental systemic infection. Thus, the sptP mutant strain of S. Enteritidis C50336 has the potential of being a safe, immunogenic vaccine against Salmonella infection.

\begin{abstract}
Abbreviations
CFU: Colony-forming units; DPI: Days post-immunization; ELISA: Enzymelinked immunosorbent assay; IL-8: Interleukin-8; LD 50 : 50\% lethal dose; $\mathrm{mLN}$ : mesenteric lymphadenitis; PBS: phosphate-buffered saline; $S$. Enteritidis: Salmonella enterica serovar Enteritidis; SI: Stimulation index; SPI-1: Salmonella pathogenicity islands 1; SptP: Salmonella protein tyrosine phosphatase; T3SS: Salmonella Type III secretion system
\end{abstract}

\section{Acknowledgments}

Not applicable.

\section{Funding}

This work was supported by the Projects of International Cooperation and Exchanges NSFC (31320103907); The Special Fund for Agro-Scientific Research in the Public Interest (201403054); The National Key Research and Development Program Special Project (2016YFD0501607); The Program for High-end talents of Yangzhou University; The Graduate Student Research and Innovation Project of Jiangsu Province (KYZZ_0368); The "Six Talent Peaks Program" of Jiangsu Province (NY-028); The Yangzhou University Science and Technology Innovation Team; Priority Academic Development Program of Jiangsu Higher Education Institutions.

\section{Availability of data and materials}

The datasets generated during and/or analysed during the current study are available from the corresponding author on reasonable request.

\section{Authors' contributions}

$Z \mathrm{Z}$ and $\mathrm{XJ}$ : the conception and design of the study. ZL, PT, YJ, XK, QL and XX: acquisition of data, analysis and interpretation of data. ZL, PT and JS: wrote the main manuscript text, prepared figures, statistical analysis and revised the manuscript. QL, XX and JS: technical, material support. XJ: final approval of the version to be submitted. All authors read and approved the final manuscript.

\section{Competing interests}

The authors declare that they have no competing interests.

\section{Consent for publication}

Not applicable.

\section{Ethics approval}

The animal experiments were all approved by the Animal Care and Ethics Committee of Yangzhou University, Yangzhou, China (Approval ID: SYXK [Su] 2012-0029).

\section{Publisher's Note}

Springer Nature remains neutral with regard to jurisdictional claims in published maps and institutional affiliations.

\section{Author details}

${ }^{1}$ Jiangsu Key Laboratory of Zoonosis, Jiangsu Co-Innovation Center for Prevention and Control of Important Animal Infectious Diseases and Zoonosis, MOA Key Laboratory of Prevention and Control of Biological Hazard Factors (Animal Origin) for Agrifood Safety and Quality, MOE Joint International Research Laboratory of Agriculture and Agri-product Safety, Yangzhou University, Yangzhou 225001, China. 'Division of Gastroenterology and Hepatology, College of Medicine, University of Illinois at Chicago, Chicago 60612, USA. ${ }^{3}$ Center for Comparative Medicine, Animal Infectious Disease Laboratory, College of Veterinary Medicine, Yangzhou University, Yangzhou 225001, China. ${ }^{4}$ Department of Anatomy and Cell Biology, Rush University Medical Center, Chicago 60612, USA.

Received: 5 October 2016 Accepted: 16 June 2017

Published online: 24 June 2017

\section{References}

1. Chai SJ, White PL, Lathrop SL, Solghan SM, Medus C, McGlinchey BM, et al. Salmonella enterica serotype Enteritidis: increasing incidence of domestically acquired infections. Clinical infectious diseases: an official publication of the Infectious Diseases Society of America. 2012;54(Suppl 5):S488-97.

2. Majowicz SE, Musto J, Scallan E, Angulo FJ, Kirk M, O'Brien SJ, et al. International collaboration on enteric disease 'Burden of illness S: the global burden of nontyphoidal Salmonella gastroenteritis. Clinical infectious diseases: an official publication of the Infectious Diseases Society of America. 2010;50(6):882-9.

3. Scallan E, Hoekstra RM, Angulo FJ, Tauxe RV, Widdowson M-A, Roy SL, et al. Foodborne illness acquired in the United States-major pathogens. Emerg Infect Dis. 2011;17(1):7-15.

4. Braden CR. Salmonella enterica serotype Enteritidis and eggs: a national epidemic in the United States. Clinical infectious diseases: an official publication of the Infectious Diseases Society of America. 2006;43(4):512-7.

5. CDC. Salmonella serotype enteritidis infections among workers producing poultry vaccine-Maine. MMWR Morb Mortal Wkly Rep 2007 Aug 31. November-December 2006;56(34):877-9.

6. Tollefson LM. Margaret Ann: antibiotic use in food animals: controlling the human health impact. J AOAC Int. 2000;83(2):245-54.

7. Whichard JM, Gay K, Stevenson JE, Joyce KJ, Cooper KL, Omondi M, et al. Human Salmonella and concurrent decreased susceptibility to quinolones and extended-spectrum cephalosporins. Emerg Infect Dis. 2007;13(11):1681-8.

8. Mastroeni P, Chabalgoity JA, Dunstan SJ, Maskell DJ, Dougan G. Salmonella: immune responses and vaccines. Vet J. 2001;161(2):132-64.

9. Geng S, Jiao X, Barrow P, Pan Z, Chen X. Virulence determinants of Salmonella Gallinarum biovar Pullorum identified by PCR signature-tagged mutagenesis and the spiC mutant as a candidate live attenuated vaccine. Vet Microbiol. 2014;168(2-4):388-94.

10. Haneda T, Okada N, Kikuchi Y, Takagi M, Kurotaki T, Miki T, et al. Evaluation of Salmonella enterica serovar Typhimurium and Choleraesuis slyA mutant strains for use in live attenuated oral vaccines. Comp Immunol Microbiol Infect Dis. 2011;34(5):399-409.

11. Bohez L, Ducatelle R, Pasmans F, Haesebrouck F, Van Immerseel F. Longterm colonisation-inhibition studies to protect broilers against colonisation with Salmonella Enteritidis, using Salmonella Pathogenicity Island 1 and 2 mutants. Vaccine. 2007;25(21):4235-43.

12. Karasova D, Sebkova A, Vrbas V, Havlickova H, Sisak F, Rychlik I. Comparative analysis of Salmonella enterica serovar Enteritidis mutants with a vaccine potential. Vaccine. 2009;27(38):5265-70.

13. Si W, Wang X, Liu H, Yu S, Li Z, Chen L, et al. Physiology, pathogenicity and immunogenicity of live, attenuated Salmonella enterica serovar Enteritidis mutants in chicks. Microb Pathog. 2015;83-84:6-11.

14. Araya DV, Quiroz TS, Tobar HE, Lizana RJ, Quezada CP, Santiviago CA, et al. Deletion of a prophage-like element causes attenuation of Salmonella enterica serovar Enteritidis and promotes protective immunity. Vaccine. 2010;28(33):5458-66.

15. Fu Y, Galan JE. A Salmonella protein antagonizes Rac-1 and Cdc42 to mediate host-cell recovery after bacterial invasion. Nature. 1999;401(6750):293-7. 
16. Murli S, Watson RO, Galan JE. Role of tyrosine kinases and the tyrosine phosphatase SptP in the interaction of Salmonella with host cells. Cell Microbiol. 2001;3(12):795-810.

17. Haraga A, Miller SI. A Salmonella enterica Serovar Typhimurium translocated leucine-rich repeat effector protein inhibits NF- B-dependent Gene expression. Infect Immun. 2003;71(7):4052-8.

18. Lin SL, Le TX, Cowen DS. SptP, a Salmonella Typhimurium type III-secreted protein, inhibits the mitogen-activated protein kinase pathway by inhibiting Raf activation. Cell Microbiol. 2003;5(4):267-75.

19. Choi HW, Brooking-Dixon R, Neupane S, Lee CJ, Miao EA, Staats HF, et al. Salmonella Typhimurium impedes innate immunity with a mast-cellsuppressing protein tyrosine phosphatase. SptP Immunity. 2013;39(6):1108-20.

20. Datsenko KA, Wanner BL. One-step inactivation of chromosomal genes in Escherichia coli K-12 using PCR products. Proc Natl Acad Sci U S A. 2000:97(12):6640-5.

21. McCormick BA. Salmonella Typhimurium attachment to human intestinal epithelial monolayers: transcellular signalling to subepithelial neutrophils. J Cell Biol. 1993;123(4):895-907.

22. Zhang X, Kelly SM, Bollen WS, Curtiss R 3rd. Characterization and immunogenicity of Salmonella Typhimurium SL1344 and UK-1 delta crp and delta cdt deletion mutants. Infect Immun. 1997:65(12):5381-7.

23. Gilles HJ. Calculation of the index of acute toxicity by the method of linear regression. Comparison with the method of "Karber and Behrens". European journal of toxicology and environmental hygiene Journal europeen de toxicologie. 1974;7(2):77-84.

24. Meng X, Meng X, Zhu C, Wang H, Wang J, Nie J, et al. The RNA chaperone $\mathrm{Hfq}$ regulates expression of fimbrial-related genes and virulence of Salmonella enterica serovar Enteritidis. FEMS Microbiol Lett. 2013;346(2):90-6.

25. Rana N, Kulshreshtha RC. Cell-mediated and humoral immune responses to a virulent plasmid-cured mutant strain of Salmonella enterica serotype gallinarum in broiler chickens. Vet Microbiol. 2006;115(1-3):156-62.

26. Brito JR, Hinton M, Stokes CR, Pearson GR. The humoral and cell mediated immune response of young chicks to Salmonella Typhimurium and S. Kedougou. The British veterinary journal. 1993;149(3):225-34.

27. Strugnell RA, Scott TA, Wang N, Yang CY, Peres N, Bedoui S, et al. Salmonella vaccines: lessons from the mouse model or bad teaching? Curr Opin Microbiol. 2014;17(17C):99-105.

28. Morpeth SC, Ramadhani HO, Crump JA. Invasive non-Typhi Salmonella disease in Africa. Clinical infectious diseases: an official publication of the Infectious Diseases Society of America. 2009:49(4):606-11.

29. Katsenos C, Anastasopoulos N, Patrani M, Mandragos C. Salmonella Enteritidis meningitis in a first time diagnosed AIDS patient: case report. Cases journal. 2008;1(1):5.

30. Gordon MA, Graham SM, Walsh AL, Wilson L, Phiri A, Molyneux E, et al. Epidemics of invasive Salmonella enterica serovar Enteritidis and S. Enterica Serovar Typhimurium infection associated with multidrug resistance among adults and children in Malawi. Clinical infectious diseases: an official publication of the Infectious Diseases Society of America. 2008;46(7):963-9.

31. Tena D, Gonzalez-Praetorius A, Bisquert J. Urinary tract infection due to non-typhoidal Salmonella: report of 19 cases. The Journal of infection. 2007:54(3):245-9.

32. Kobayashi H, Hall GS, Tuohy MJ, Knothe U, Procop GW, Bauer TW. Bilateral periprosthetic joint infection caused by Salmonella enterica serotype Enteritidis, and identification of Salmonella sp using molecular techniques. International journal of infectious diseases: IJID: official publication of the International Society for Infectious Diseases. 2009;13(6):e463-6.

33. Mutlu H, Babar J, Maggiore PR: Extensive Salmonella Enteritidis endocarditis involving mitral, tricuspid valves, aortic root and right ventricular wall. Journal of the American Society of Echocardiography: official publication of the American Society of Echocardiography 2009, 22(2):210. e1-3.

34. Ghosh TS, Vogt RL. Cluster of invasive salmonellosis cases in a federal prison in Colorado. Am J Infect Control. 2006;34(6):348-50.

35. Khan SA, Stratford R, Wu T, Mckelvie N, Bellaby T, Hindle Z, et al. Salmonella Typhi and S-Typhimurium derivatives harbouring deletions in aromatic biosynthesis and Salmonella Pathogenicity Island-2 (SPI-2) genes as vaccines and vectors. Vaccine. 2003;21(5-6):538-48.

36. Coombes BK, Coburn BA, Potter AA, Gomis S, Mirakhur K, Li Y, et al. Analysis of the contribution of Salmonella pathogenicity islands 1 and 2 to enteric disease progression using a novel bovine ileal loop model and a murine model of infectious enterocolitis. Infect Immun. 2005:73(11):7161-9.
37. Boyen F, Pasmans F, Van Immerseel F, Morgan E, Botteldoorn N, Heyndrickx M, et al. A limited role for SsrA/B in persistent Salmonella Typhimurium infections in pigs. Vet Microbiol. 2008;128(3-4):364-73.

38. Dieye $Y$, Ameiss K, Mellata M, Curtiss R 3rd. The Salmonella Pathogenicity Island (SPI) 1 contributes more than SPI2 to the colonization of the chicken by Salmonella enterica serovar Typhimurium. BMC Microbiol. 2009;9(1):1-14.

39. Karasova D, Sebkova A, Havlickova H, Sisak F, Volf J, Faldyna M, et al. Influence of 5 major Salmonella pathogenicity islands on NK cell depletion in mice infected with Salmonella enterica serovar Enteritidis. BMC Microbiol. 2010;10:75.

40. Matulova M, Havlickova H, Sisak F, Rychlik I. Vaccination of chickens with Salmonella Pathogenicity Island (SPI) 1 and SPI2 defective mutants of Salmonella enterica serovar Enteritidis. Vaccine. 2012;30(12):2090-7.

41. Collins FM, Scott MT. Effect of Corynebacterium Parvum treatment on the growth of Salmonella Enteritidis in mice. Infect Immun. 1974;9(5):863-9.

42. Berndt A, Wilhelm A, Jugert C, Pieper J, Sachse K, Methner U. Chicken cecum immune response to Salmonella enterica serovars of different levels of invasiveness. Infect Immun. 2007;75(12):5993-6007.

43. Haraga A, Ohlson MB, Miller SI. Salmonellae interplay with host cells. Nat Rev Microbiol. 2008;6(1):53-66

44. Cirillo DM, Valdivia RH, Monack DM, Falkow S. Macrophage-dependent induction of the Salmonella pathogenicity island 2 type III secretion system and its role in intracellular survival. Mol Microbiol. 1998;30(1):175-88.

\section{Submit your next manuscript to BioMed Central and we will help you at every step:}

- We accept pre-submission inquiries

- Our selector tool helps you to find the most relevant journal

- We provide round the clock customer support

- Convenient online submission

- Thorough peer review

- Inclusion in PubMed and all major indexing services

- Maximum visibility for your research

Submit your manuscript at www.biomedcentral.com/submit
) Biomed Central 